\title{
Some elementary explicit bounds for two mollifications of the Moebius function
}

\author{
O. Ramaré
}

May 15, 2013

\begin{abstract}
We prove that the sum $\sum_{\left\{\begin{array}{c}d \leq x, \\ (d, r)=1\end{array}\right.} \mu(d) / d^{1+\varepsilon}$ is bounded by $1+\varepsilon$, uniformly in $x \geq 1, r$ and $\varepsilon>0$. We prove a similar estimate for the quantity $\sum_{\left\{\begin{array}{c}d \leq x, \\ (d, r)=1\end{array}\right.} \mu(d) \log (x / d) / d^{1+\varepsilon}$. When $\varepsilon=0, r$ varies between 1 and a hundred, and $x$ is below a million, this sum is non-negative and this raises the question as to whether it is non-negative for every $x$.
\end{abstract}

\section{Introduction and results}

Our first result is the following:

Theorem 1.1. When $r \geq 1$ and $\varepsilon \geq 0$, we have

$$
\left|\sum_{\substack{d \leq x,(d, r)=1}} \frac{\mu(d)}{d^{1+\varepsilon}}\right| \leq 1+\varepsilon .
$$

This Lemma generalizes the estimate of [5, Lemme 10.2] which corresponds to the case $\varepsilon=0$. This generalization is not straightforward at all and requires a change of proof. The case $\varepsilon=0$ and $r=1$ is classical. The parameter $\varepsilon$ that is being introduced induces some flexibility useful when applying Rankin's method (devised in [8]). As it turns out, we can do somewhat better concerning the lower bound, and we prove that

$$
-\frac{11}{15}(1+\varepsilon) \leq \sum_{\substack{d \leq x \\(d, r)=1}} \frac{\mu(d)}{d^{1+\varepsilon}} .
$$

We ran computations covering the range $1 \leq x \leq 10^{6}$ and $1 \leq r \leq 100$ with $\varepsilon=0$; we found that the lowest lower bound was met at $x=13$ and $r=1$. This raises the following question:

AMS Classification: 11N37, 11Y35, secondary : 11A25

Keywords: Explicit estimates, Moebius function 
Question 1. It is true that

$$
\sum_{\substack{d \leq x,(d, r)=1}} \frac{\mu(d)}{d} \geq-2323 / 30030
$$

See section 2 for a very preliminary result in this direction.

We proceed by proving the following more involved form:

Theorem 1.2. When $r \geq 1$ and $1.38 \geq \varepsilon \geq 0$, we have

$$
\left|\sum_{\substack{d \leq x,(d, r)=1}} \frac{\mu(d)}{d^{1+\varepsilon}} \log \frac{x}{d}\right| \leq 1.4+4.7 \varepsilon+3.3 \varepsilon^{2}+(1+\varepsilon) \frac{r^{1+\varepsilon}}{\phi_{1+\varepsilon}(r)} x^{\varepsilon}
$$

where

$$
\frac{r^{1+\varepsilon}}{\phi_{1+\varepsilon}(r)}=\prod_{p \mid r} \frac{p^{1+\varepsilon}}{p^{1+\varepsilon}-1} .
$$

The dependence in $r$ is optimal as seen by taking for $r$ the product of every primes not more than $\sqrt{x}$. The proof is again unbalanced with respect to the upper and the lower bound, and we prove a somewhat better lower bound:

$$
-\left(1.434+4.992 \varepsilon+3.558 \varepsilon^{2}\right) \leq \sum_{\substack{d \leq x,(d, r)=1}} \frac{\mu(d)}{d^{1+\varepsilon}} \log \frac{x}{d} .
$$

I expect the factor $x^{\varepsilon}$ in the upper bound to be a blemish; however, the (limited) numerical verifications we ran suggest that the factor $r^{1+\varepsilon} / \phi_{1+\varepsilon}(r)$ cannot be omitted even if the condition $r \leq x$ is added (this condition often appears in practice). It should be added that it is not difficult to prove that

$$
\sum_{d \leq x} \frac{\mu(d)}{d} \log \frac{x}{d} \sim 1 \quad(x \rightarrow \infty)
$$

which means that one cannot expect an arbitary small constant in the right hand side of the inequality given in Theorem 1.2. We have checked that

$$
0 \leq \sum_{\substack{d \leq x,(d, r)=1}} \frac{\mu(d)}{d} \log \frac{x}{d} \leq \frac{r}{\phi(r)}+0.007 \quad\left(x \leq 10^{6}, 1 \leq r \leq 100\right)
$$

(where $x$ is a real number and not especially an integer) and all these maxima were in fact very close to $r / \phi(r)$. These computations raise two questions:

Question 2. Is it true that

$$
\sum_{\substack{d \leq x,(d, r)=1}} \frac{\mu(d)}{d} \log \frac{x}{d} \geq 0, \quad(x \geq 1, r \geq 1) \quad \text { ? }
$$


Question 3. Is it true that

$$
\sum_{\substack{d \leq x,(d, r)=1}} \frac{\mu(d)}{d} \log \frac{x}{d} \leq \frac{r}{\phi(r)}+1, \quad(x \geq 1, r \geq 1) \quad ?
$$

In both these questions, $x$ is only assumed to be a positive real number. On recalling what happens in the case of Turán's conjecture on the summatory function of the Liouville function divided by its argument, see [2], we believe that the answer to the first question is no. The sum is however less likely to be very erratical because of the smoothing factor, a factor that is absent in Turán's problem. In direction of these conjecture, we note the following formula

$$
\int_{1}^{\infty} \sum_{\substack{d \leq x,(d, r)=1}} \frac{\mu(d)}{d} \log \frac{x}{d} \frac{d x}{x^{s+1}}=\frac{r^{1+s}}{\phi_{1+s}(r)} \frac{1}{s^{2} \zeta(1+s)}
$$

from which we easily deduce (on taking $s=\varepsilon>0$ and letting $\varepsilon$ go to infinity) that

$$
\limsup _{x} \sum_{\substack{d \leq x,(d, r)=1}} \frac{\mu(d)}{d} \log \frac{x}{d} \geq \frac{r}{\phi(r)} .
$$

We discuss some related points in the last section.

\section{Notation}

We use here the notation $h=\mathcal{O}^{*}(k)$ to mean that $|h| \leq k$. We denote by $\tau(m)$ the number of (positive) divisors of $m$, and by $(a, b)$ the gcd of $a$ and $b$. For $\varepsilon \geq 0$ and $r \geq 1$ any natural squarefree number, we define two functions. The first one is alternatively defined by

$$
f_{r, \varepsilon}(n)=\sum_{\substack{\ell \mid n,(\ell, r)=1}} \frac{\mu(\ell)}{\ell^{\varepsilon}} \tau(n / \ell)
$$

or, in multiplicative form, by:

$$
f_{r, \varepsilon}(n)=\prod_{\substack{\nu \\ p^{\prime} \| n, p \nmid r}}\left(\nu+1-\frac{\nu}{p^{\varepsilon}}\right) \prod_{\substack{\nu \\ p^{\nu} \| n, p \mid r}}(\nu+1) .
$$

We easily determine its Dirichlet series: $\sum_{n \geq 1} f_{r, \varepsilon}(n) / n^{s}=\zeta(s)^{2} / \zeta(s+\varepsilon)$. We shall further write

$$
f_{r, \varepsilon}(n)=\mathbb{1} \star g_{r, \varepsilon}(n)
$$


where the function $g_{r, \varepsilon}$ has the essential property of being non-negative and is being defined by:

$$
g_{r, \varepsilon}(n)=\sum_{\substack{\ell \mid n,(\ell, r)=1}} \frac{\mu(\ell)}{\ell^{\varepsilon}} \geq 0 .
$$

\section{Thanks}

Sincere thanks are due to the careful referee who has checked our computations and indeed has rooted out several mistakes.

\section{$2 \quad$ Verifying Theorem 1.1 for small values}

We study what happens for small values here. The proof is pedestrian and painful, but I have not seen any way to avoid it, or to present it in a more general frame.

We study the following quantity:

$$
m_{0}(r, x)=\sum_{\substack{d \leq x \\(d, r)=1}} \frac{\mu(d)}{d^{1+\varepsilon}} .
$$

Lemma 2.1. When $x<10$ and $\varepsilon \geq 0$, we have $-1 / 30 \leq m_{0}(r, x) \leq 1$.

Proof. The sum we consider reads

$$
1-\frac{h(2)}{2^{1+\varepsilon}}-\frac{h(3)}{3^{1+\varepsilon}}-\frac{h(5)}{5^{1+\varepsilon}}+\frac{h(6)}{6^{1+\varepsilon}}-\frac{h(7)}{7^{1+\varepsilon}}
$$

where $h$ is the characteristic function of the integers $\leq x$ that are coprime with $r$. The minimum is clearly

$$
1-\frac{1}{2^{1+\varepsilon}}-\frac{1}{3^{1+\varepsilon}}-\frac{1}{5^{1+\varepsilon}}
$$

which is minimal when $\varepsilon=0$. This is the $-1 / 30$. The maximum contains the summand 1 . If the summand $1 / 6^{1+\varepsilon}$ is present, then so is the summand $-1 / 2^{1+\varepsilon}$. This concludes the proof.

\section{Auxiliaries}

Lemma 3.1. When $\varepsilon \geq 0$, we have

$$
\sum_{h \leq H} h^{\varepsilon}=\frac{H^{1+\varepsilon}}{1+\varepsilon}+\mathcal{O}^{*}\left(H^{\varepsilon}\right) .
$$

This is also $\leq H^{1+\varepsilon}$. When $H$ is an integer, we have $\sum_{h \leq H} h^{\varepsilon} \geq \frac{H^{1+\varepsilon}}{1+\varepsilon}$. 
Proof. Indeed, when $\varepsilon>0$, a summation by parts gives us directly

$$
\begin{aligned}
\sum_{h \leq H} h^{\varepsilon} & =\sum_{h \leq H} \varepsilon \int_{0}^{h} d t / t^{1-\varepsilon}=\varepsilon \int_{0}^{H} \sum_{t<h \leq H} 1 d t / t^{1-\varepsilon} \\
& =\varepsilon \int_{0}^{H}(H-t) d t / t^{1-\varepsilon}+\mathcal{O}^{*}\left(H^{\varepsilon}\right) .
\end{aligned}
$$

We proceed by continuity to cover the case $\varepsilon=0$. When $H$ is an integer, a comparison to an integral gives the result.

Lemma 3.2. For $L>1$, we have

$$
\sum_{n \leq L} f_{r, \varepsilon}(n) \leq L \sum_{\ell \leq L} g_{r, \varepsilon}(\ell) / \ell
$$

Proof. We recall (4) and write, since $g_{r, \varepsilon} \geq 0$

$$
\sum_{n \leq L} f_{r, \varepsilon}(n)=\sum_{k m \leq L} g_{r, \varepsilon}(m) \leq L \sum_{m \leq L} g_{r, \varepsilon}(m) / m .
$$

The Lemma follows readily.

Lemma 3.3. For every integer $n$ and any $\varepsilon \geq 0$, we have

$$
g_{1, \varepsilon}(\ell) \leq \sum_{m n=\ell} g_{1, \varepsilon / 2}(m) g_{1, \varepsilon / 2}(n)
$$

Proof. We check that, when $\alpha \geq 1$ is an integer and $p$ a prime number,

$$
\begin{aligned}
g_{1, \varepsilon}\left(p^{\alpha}\right) & =1-\frac{1}{p^{\varepsilon}}=1-\frac{1}{p^{\varepsilon / 2}}+\frac{1}{p^{\varepsilon / 2}}\left(1-\frac{1}{p^{\varepsilon / 2}}\right) \\
\leq \leq g_{1, \varepsilon / 2}\left(p^{\alpha}\right) g_{1, \varepsilon / 2}(1)+g_{1, \varepsilon / 2}(1) g_{1, \varepsilon / 2}\left(p^{\alpha}\right) & \leq \sum_{0 \leq \beta \leq \alpha} g_{1, \varepsilon / 2}\left(p^{\alpha-\beta}\right) g_{1, \varepsilon / 2}\left(p^{\beta}\right) .
\end{aligned}
$$

We conclude by invoking the multiplicativity of $g_{1, \varepsilon / 2}$.

Lemma 3.4. We have when $L \geq 7.2$,

$$
\sum_{p \leq L} \frac{\log p}{p-1} \leq \log L
$$

Proof. We cite $[9,(2.8)]$ :

$$
\sum_{p \leq L} \frac{\log p}{p} \leq \log L-\gamma-\sum_{p \geq 2} \frac{\log p}{p(p-1)}+\frac{1}{2 \log L}, \quad(L \geq 319)
$$


from which we deduce, for $L \geq 319$,

$$
\sum_{p \leq L} \frac{\log p}{p-1} \leq \log L-\gamma+\frac{1}{2 \log L} .
$$

A simple GP script shows that

$$
\sum_{p \leq L} \frac{\log p}{p-1} \leq \log L
$$

when $1000 \geq L \geq 7.2$, and the reader will conclude readily.

Lemma 3.5. We have, when $L \geq 1$ and $\varepsilon \geq 0$,

$$
\sum_{\ell \leq L} g_{1, \varepsilon}(\ell) / \ell \leq L^{\varepsilon} .
$$

Proof. Verifying the stated inequality for $1 \leq L<8$ is (tedious but) easy, hence we can now assume that $L \geq 8$. We readily find that the sum in question is not more than

$$
T=\prod_{p \leq L} \frac{1-p^{-1-\varepsilon}}{1-p^{-1}}=\exp \sum_{p \leq L} \log \left(1+\frac{1-p^{-\varepsilon}}{p-1}\right) .
$$

We apply $\log (1+x) \leq x$ for non-negative $x$ and $1-p^{-\varepsilon} \leq \varepsilon \log p$ to get, when $L \geq 8$,

$$
T \leq \exp \varepsilon \sum_{p \leq L} \frac{\log p}{p-1} \leq L^{\varepsilon}
$$

by invoking Lemma 3.4 .

Lemma 3.6. We have, when $L \geq 1, r \geq 1$ and $\varepsilon \geq 0$,

$$
\sum_{\ell \leq L} g_{r, \varepsilon}(\ell) / \ell \leq \frac{r^{1+\varepsilon}}{\phi_{1+\varepsilon}(r)} L^{\varepsilon} .
$$

Proof. We use the notation $d \mid r^{\infty}$ to say that each prime factor of $d$ divides $r$. We write

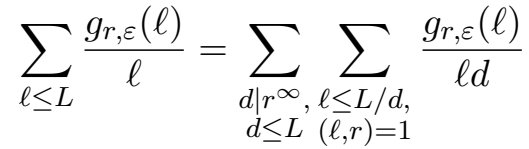

$$
\begin{aligned}
& \leq L^{\varepsilon} \sum_{d \mid r^{\infty}} \frac{1}{d^{1+\varepsilon}}=L^{\varepsilon} \frac{r^{1+\varepsilon}}{\phi_{1+\varepsilon}(r)}
\end{aligned}
$$

by Lemma 3.5. The Lemma follows readily. 


\section{Lemma 3.7.}

$$
\sum_{m \leq M} m^{\varepsilon} \tau(m)=\frac{M^{1+\varepsilon}}{1+\varepsilon}\left(\log M+2 \gamma-\frac{1}{1+\varepsilon}\right)+\mathcal{O}^{*}\left(0.961(1+2 \varepsilon) M^{\frac{1}{2}+\varepsilon}\right)
$$

Proof. We recall part of $[1$, Theorem 1.1]:

$$
\sum_{m \leq t} \tau(m)=t \log t+(2 \gamma-1) t+\mathcal{O}^{*}(0.961 \sqrt{t}), \quad(t \geq 1) .
$$

Since $(t \log t+(2 \gamma-1) t) / \sqrt{t}$ is seen to vary between -0.681 and 0.155 when $t$ varies between 0 and 1 , this estimate is also valid for $t>0$. We use summation by parts and find that

$$
\begin{aligned}
\sum_{m \leq M} m^{\varepsilon} \tau(m) & =M^{\varepsilon} \sum_{m \leq M} \tau(m)-\varepsilon \int_{0}^{M} \sum_{m \leq t} \tau(m) d t / t^{1-\varepsilon} \\
& =M^{1+\varepsilon}(\log M+2 \gamma-1)+\mathcal{O}^{*}\left(0.961 M^{\frac{1}{2}+\varepsilon}\right) \\
& -\varepsilon \int_{0}^{M}(\log t+2 \gamma-1) t^{\varepsilon} d t+\mathcal{O}^{*}\left(0.961 \varepsilon \int_{0}^{M} t^{\varepsilon-1 / 2} d t\right) \\
& =\frac{M^{1+\varepsilon}}{1+\varepsilon}\left(\log M+2 \gamma-\frac{1}{1+\varepsilon}\right)+\mathcal{O}^{*}\left(0.961(1+2 \varepsilon) M^{\frac{1}{2}+\varepsilon}\right) .
\end{aligned}
$$

Lemma 3.8. We have, when $n \geq 2$,

$$
g_{r, \varepsilon}(n) \leq 1-\frac{\mathbb{1}_{(n, r)=1} \mu^{2}(n)}{n^{\varepsilon}} .
$$

Proof. Indeed, we verify that $(1-a)(1-b) \leq(1-a b)$ when $0 \leq a, b \leq 1$. The Lemma readily follows by recursion on the number of prime factors of $n$.

\section{Some lemmas on squarefree numbers}

Here is a Lemma from [4]:

Lemma 4.1. We have, for $D \geq 1664$

$$
\sum_{d \leq D} \mu^{2}(d)=\frac{6 D}{\pi^{2}}+\mathcal{O}^{*}(0.1333 \sqrt{D})
$$

In particular, this is not more than $0.62 D$ when $D \geq 1700$. 
Lemma 4.2. We have

$$
\sum_{d \leq x} \mu^{2}(d) / \sqrt{d} \leq 1.33 \sqrt{x}, \quad(x \geq 1) .
$$

If we are ready to assume larger, we would not save much since the best constant one can get is $12 / \pi^{2}=1.215+\mathcal{O}^{*}(0.001)$.

Proof. We use PARI/GP see [7] and the following script:

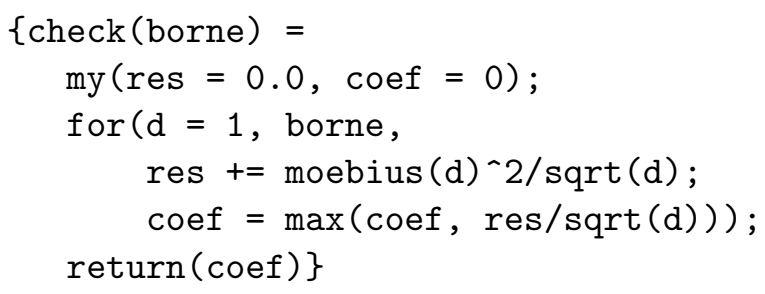

It is then almost immediate to check our result when $x \leq 10^{7}$, despite the lack of refinement of the script proposed. For larger values, we use a summation by parts together with Lemma 4.1 .

Lemma 4.3. We have

$$
\sum_{d \leq x} \mu^{2}(d) \leq \frac{11}{15} x, \quad(x \geq 9) .
$$

We note that $11 / 15=0.7333 \ldots$ while the asymptotically best constant is rather lower, namely $6 / \pi^{2}=0.607927 \ldots$ Reaching $73 / 115=0.63478 \ldots$ already requires to take $x \geq 75$, and this means we would have to handle the possible divisibility by 21 primes in section 2 . This is out of reach of the simple-minded method we have at our disposal.

Proof. We use PARI/GP see [7] and the following script:

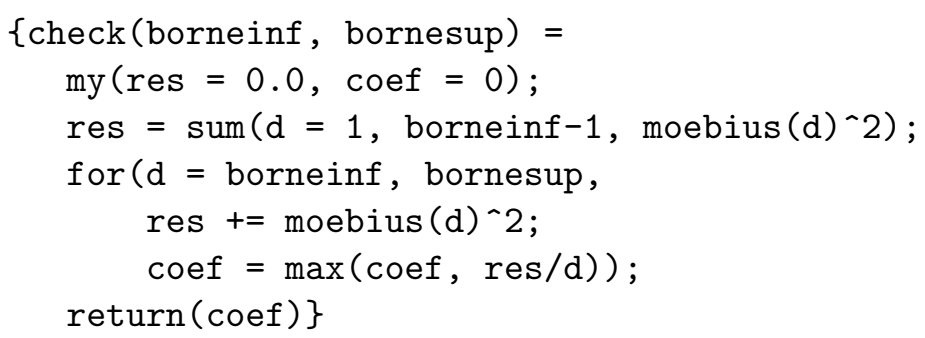

It is then almost immediate to check our result when $x \leq 10^{7}$, despite the lack of refinement of the script proposed. For larger values, the result is an immediate consequence of Lemma 4.1. 


\section{$5 \quad$ Proof of Theorem 1.1}

Lemma 2.1 establishes Theorem 1.1 when $x<10$, so we may assume $x \geq 10$. We further may restrict our attention to integer values of $x$. We start with

$$
S_{0}=\sum_{n \leq x} n^{\varepsilon} g_{r, \varepsilon}(n)=\sum_{n \leq x} \sum_{\substack{d \mid n,(d, r)=1}} \mu(d)(n / d)^{\varepsilon} .
$$

Using the first expression yields $0 \leq S_{0}$ as well as

$$
S_{0} / x^{\varepsilon} \leq 1+\sum_{2 \leq n \leq x}\left(g_{r, \varepsilon}(n)+\frac{\mathbb{1}_{(n, r)=1} \mu^{2}(n)}{n^{\varepsilon}}\right)-\sum_{\substack{2 \leq n \leq x, n, r)=1}} \frac{\mu^{2}(n)}{n^{\varepsilon}}
$$

Each summand in the second sum is bounded above by 1 by Lemma 3.8. We get

$$
0 \leq S_{0} / x^{\varepsilon} \leq x-\sum_{\substack{2 \leq n \leq x,(n, r)=1}} \frac{\mu^{2}(n)}{n^{\varepsilon}} .
$$

Let us write the second expression for $S_{0}$ :

$$
S_{0}=\sum_{\substack{d \leq x,(d, r)=1}} \mu(d) \sum_{m \leq x / d} m^{\varepsilon} .
$$

We employ Lemma 3.1; we treat the case $d=1$ separately for the lower bound to reach

$$
\begin{gathered}
\frac{x^{1+\varepsilon}}{1+\varepsilon} \sum_{\substack{d \leq x,(d, r)=1}} \frac{\mu(d)}{d^{1+\varepsilon}}-x^{\varepsilon} \sum_{\substack{2 \leq d \leq x,(d, r)=1}} \mu^{2}(d) d^{-\varepsilon} \leq S_{0} \\
\leq \frac{x^{1+\varepsilon}}{1+\varepsilon} \sum_{\substack{d \leq x,(d, r)=1}} \frac{\mu(d)}{d^{1+\varepsilon}}+x^{\varepsilon} \sum_{\substack{d \leq x,(d, r)=1}} \mu^{2}(d) d^{-\varepsilon} .
\end{gathered}
$$

The lower bound requires $x$ to be an integer, but not the upper bound. We rewite the above as

$$
S_{0}-x^{\varepsilon} \sum_{\substack{d \leq x,(d, r)=1}} \mu^{2}(d) d^{-\varepsilon} \leq \frac{x^{1+\varepsilon}}{1+\varepsilon} \sum_{\substack{d \leq x,(d, r)=1}} \frac{\mu(d)}{d^{1+\varepsilon}} \leq S_{0}+x^{\varepsilon} \sum_{\substack{2 \leq d \leq x \\(d, r)=1}} \mu^{2}(d) d^{-\varepsilon} .
$$

By conjugating both estimates, we get,

$$
-x^{\varepsilon} \sum_{\substack{d \leq x,(d, r)=1}} \mu^{2}(d) d^{-\varepsilon} \leq \frac{x^{1+\varepsilon}}{1+\varepsilon} \sum_{\substack{d \leq x,(d, r)=1}} \frac{\mu(d)}{d^{1+\varepsilon}} \leq x^{1+\varepsilon} .
$$


The right hand side is easily handled. We use Lemma 4.3 for the left hand side via, when $x \geq 9$ :

$$
\sum_{\substack{d \leq x,(d, r)=1}} \mu^{2}(d) d^{-\varepsilon} \leq \sum_{d \leq x} \mu^{2}(d) \leq \frac{11}{15} x .
$$

By conjugating both estimates, we get

$$
-\frac{11}{15}(1+\varepsilon) \leq \sum_{\substack{d \leq x \\(d, r)=1}} \frac{\mu(d)}{d^{1+\varepsilon}} \leq 1+\varepsilon . \quad(x \geq 9)
$$

Theorem 1.1 is proved.

\section{Proof of Theorem 1.2}

The proof relies on two ways of writing the sum

$$
S_{1}=\sum_{n \leq x} n^{\varepsilon} f_{r, \varepsilon}(n)=\sum_{n \leq x} \sum_{\substack{d \mid n,(d, r)=1}} \mu(d)(n / d)^{\varepsilon} \tau(n / d) .
$$

The first form shows that $0 \leq S_{1} \leq x^{1+2 \varepsilon} r^{1+\varepsilon} / \phi_{1+\varepsilon}(r)$ by combining Lemma 3.2 together with Lemma 3.6. Let us write this sum differently:

$$
S_{1}=\sum_{\substack{d \leq x,(d, r)=1}} \mu(d) \sum_{m \leq x / d} m^{\varepsilon} \tau(m)
$$

and we use Lemma 3.7 to reach

$S_{1}=\frac{x^{1+\varepsilon}}{1+\varepsilon} \sum_{\substack{d \leq x,(d, r)=1}} \frac{\mu(d)}{d^{1+\varepsilon}}\left(\log \frac{x}{d}+2 \gamma-\frac{1}{1+\varepsilon}\right)+\mathcal{O}^{*}\left(0.961 \times 1.33(1+2 \varepsilon) x^{1+\varepsilon}\right)$

since $\sum_{d \leq x} \mu^{2}(d) / \sqrt{d} \leq 1.33 \sqrt{x}$ by Lemma 4.2 . We set

$$
\alpha=2 \gamma-\frac{1}{1+\varepsilon} \in[0,1] .
$$

All of that amounts to:

$$
\begin{aligned}
S_{1} & =\frac{x^{1+\varepsilon}}{1+\varepsilon} \sum_{\substack{d \leq x,(d, r)=1}} \frac{\mu(d)}{d^{1+\varepsilon}}\left(\log \frac{x}{d}+\alpha\right)+\mathcal{O}^{*}\left(1.279(1+2 \varepsilon) x^{1+\varepsilon}\right) \\
& =S_{1}^{*}+\alpha S_{0}+\mathcal{O}^{*}\left(1.279(1+2 \varepsilon) x^{1+\varepsilon}\right)
\end{aligned}
$$


say. We thus have

$$
-1.279(1+2 \varepsilon) x^{1+\varepsilon} \leq S_{1}^{*}+\alpha S_{0} \leq 1.279(1+2 \varepsilon) x^{1+\varepsilon}+x^{1+2 \varepsilon} \frac{r^{1+\varepsilon}}{\phi_{1+\varepsilon}(r)} .
$$

We use (10) and Lemma 2.1, and reach

$$
-1.279(1+2 \varepsilon)-\alpha \leq x^{-1-\varepsilon} S_{1}^{*} \leq 1.279(1+2 \varepsilon)+\frac{11}{15} \alpha+x^{\varepsilon} \frac{r^{1+\varepsilon}}{\phi_{1+\varepsilon}(r)} .
$$

We use $\alpha \leq 2 \gamma-1+\varepsilon$. This gives

$$
\begin{aligned}
-1.434-4.992 \varepsilon-3.558 \varepsilon^{2} & \leq \sum_{\substack{d \leq x,(d, r)=1}} \frac{\mu(d)}{d^{1+\varepsilon}} \log \frac{x}{d} \\
& \leq 1.393+4.684 \varepsilon+3.292 \varepsilon^{2}+(1+\varepsilon) \frac{x^{\varepsilon} r^{1+\varepsilon}}{\phi_{1+\varepsilon}(r)} .
\end{aligned}
$$

Since $x^{\varepsilon} r^{1+\varepsilon} / \phi_{1+\varepsilon}(r) \geq 1$, we check that the right hand side is larger than minus times the left hand side. Theorem 1.2 follows.

\section{$7 \quad$ A generalization and a remark}

It is not difficult to get along these lines the following Lemma:

Lemma 7.1. When $r \geq 1$ and $k \geq 1$, we have

$$
\sum_{\substack{d \leq x,(d, r)=1}} \frac{\mu(d)}{d^{1+\varepsilon}} \log ^{k} \frac{x}{d} \ll_{k}\left(\frac{r}{\phi(r)}\right)^{k}(\log x)^{k-1} .
$$

Such quantities appear for instance in [10] where cases $k=0$ and $k=1$ are used, while case $k=2$ is evaluated (there is a main term), but all with no coprimality conditions (i.e. $r=1$ ) and no $\varepsilon$. The reader will find in $[3$, Chapter 1] the evaluation of case $k=3, r=1$ and $\varepsilon=0$. [6] also pertains to these quantities.

Proof. Indeed, we first prove that

$$
\sum_{n \leq x} \sum_{\substack{d \mid n,(d, r)=1}} \mu(d)(n / d)^{\varepsilon} \tau_{k+1}(n / d) \ll\left(\frac{r}{\phi(r)}\right)^{k} x(\log x)^{k-1} .
$$

We then continue as in section 6 .

Here is a surprising elementary consequence. 
Lemma 7.2. For any $c>0$, we have

$$
\sum_{\substack{d \leq x,(d, r)=1}} \frac{\mu(d)}{d}-x^{\varepsilon} \sum_{\substack{d \leq x,(d, r)=1}} \frac{\mu(d)}{d^{1+\varepsilon}} \ll_{c} \varepsilon \frac{r}{\phi(r)}
$$

provided that $0 \leq \varepsilon \leq c(\log x)^{-1}$.

Proof. It is enough to consider

$$
\int_{0}^{\varepsilon} \sum_{\substack{d \leq x,(d, r)=1}} \frac{\mu(d) x^{\eta}}{d^{1+\eta}} \log (x / d) d \eta \ll \varepsilon \frac{r}{\phi(r)} .
$$

\section{References}

[1] D. Berkane, O. Bordellès, and O. Ramaré. Explicit upper bounds for the remainder term in the divisor problem. Math. of Comp., 81(278):10251051, 2012.

[2] P. Borwein, R. Ferguson, and M.J. Mossinghoff. Sign changes in sums of the Liouville function. Math. Comp., 77(263):1681-1694, 2008.

[3] K. Chandrasekharan. Arithmetical functions. Die Grundlehren der mathematischen Wissenschaften, Band 167. Springer-Verlag, New York, 1970.

[4] H. Cohen and F. Dress. Estimations numériques du reste de la fonction sommatoire relative aux entiers sans facteur carré. Prépublications mathématiques d'Orsay: Colloque de théorie analytique des nombres, Marseille, pages 73-76, 1988.

[5] A. Granville and O. Ramaré. Explicit bounds on exponential sums and the scarcity of squarefree binomial coefficients. Mathematika, 43(1):73$107,1996$.

[6] A. Kienast. Über die Äquivalenz zweier Ergebnisse der analytischen Zahlentheorie. Mathematische Annalen, 95:427-445, 1926. 10.1007/BF01206619.

[7] The PARI Group, Bordeaux. PARI/GP, version 2.5.2, 2011. http: //pari.math.u-bordeaux.fr/.

[8] R.A. Rankin. The difference between consecutive prime numbers. $J$. Lond. Math. Soc., 13:242-247, 1938. 
[9] J.B. Rosser and L. Schoenfeld. Approximate formulas for some functions of prime numbers. Illinois J. Math., 6:64-94, 1962.

[10] A. Selberg. An elementary proof of the prime-number theorem. Ann. Math., 50(2):305-313, 1949. 